\section{Authors' response to: Epithelial mesenchymal transition (EMT) in small airways of COPD patient}

We understand the reservations highlighted by Sohal and Walters in their letter in response to our recent work published. ${ }^{1}$

In their letter, Sohal and Walters argue that E-cadherin and ZO-1 are absent in our immunohistochemistry analysis of small bronchi of smokers and COPD patients, and that if disappeared completely epithelium would fall apart. ${ }^{1}$ In our study, we detected downregulation of E-cadherin and ZO-1 as well as a change of intercellular and apical distribution to diffuse cytoplasmic redistribution (figure 3B; in our work published in Thorax on 7 January 2013), ${ }^{2}$ but not complete absence. Accordingly, previous observations in bronchial epithelium from asthmatics, smokers and smokers with COPD also showed absence of ZO-1 and decrease of E-cadherin with no detached epithelium, ${ }^{3-5}$ which was attributed as part of the epithelial mesenchymal transition (EMT) process in large airways from asthmatics, ${ }^{3}$ and small airways in smokers with or without COPD. In this regard, it is known that there are a large number of proteins (not only E-cadherin and ZO-1), which form the junctional complex, composed of tight and adherent junctions that are differentially downregulated in small airway epithelium, ${ }^{4}$ and that although dysregulated, altogether would prevent the epithelium from falling apart.

We agree with Sohal and Walters with respect to the importance of the reticular basement membrane $(\mathrm{Rbm})$ fragmentation as a key process of mesenchymal cell migration from the airway epithelium to submucosa. In fact, we would like to recognise the presence of Rbm fragmentation in figure 3 of our recent paper. ${ }^{2}$ However, Rbm fragmentation appears to be more evident and important in large airways, where basement membrane is thicker, than in small airways where basement membrane is sometimes difficult to observe under light microscopy $(\times 1000)$.

In their recent paper, Sohal and Walters showed double-stain for cytokeratin-(s) and the 'EMT marker' S100A4 in large airway epithelium and $\mathrm{Rbm}$ that may indicate EMT, ${ }^{6}$ although some pictures showed seem saturated. ${ }^{7}$ Although immunohistochemical analysis is an appropriate technique to study protein distribution, this is not always reliable for quantitative purposes. In our view, it is mandatory to perform a multiple technical approach to provide consistent results such as immunohistochemistry, immunofluorescence, real-time RT-PCR and protein array techniques used in our study. ${ }^{2}$

Taken together, we agree with Sohal and Walters that EMT is an important remodelling process in COPD and that we must consider this process in large and small airways for a better understanding of the disease process.

\section{Javier Milara, ${ }^{1,2,3}$ Teresa Peiró, ${ }^{3}$ Adela Serrano, ${ }^{3}$} Julio Cortijo ${ }^{1,3,4}$

${ }^{1}$ Clinical Research Unit (UIC), University General

Hospital Consortium, Valencia, Spain

${ }^{2}$ Department of Biotechnology, Universidad Politécnica de Valencia, Valencia, Spain

${ }^{3}$ Research Foundation of General Hospital of Valencia, Valencia, Spain

${ }^{4}$ Department of Pharmacology, University of Valencia, Valencia, Spain

Correspondence to Dr Javier Milara, Clinical Research Unit (UIC), University General Hospital
Consortium, Valencia, Spain. Av tres cruces s/n, Valencia E-46014, Spain; xmilara@hotmail.com

Contributors This work was supported by grants SAF2011-26443 (JC), FIS CP11/00293(JM), CIBERES (CB06/06/0027), ADE10/00020 (Spanish government), ACIF/2010/114 (TP) and research grants from Regional Government (Prometeo/2008/045, 'Generalitat Valenciana'. Support from the CENIT programme (Spanish Government) was obtained.

Competing interests None.

Patient consent Obtained.

Ethics approval The Human Research Ethics Committee of University General Hospital Consortium (Valencia).

Provenance and peer review Not commissioned; internally peer reviewed.

To cite Milara J, Peiró T, Serrano A, et al. Thorax 2013;68:784.

Received 25 February 2013

Accepted 28 February 2013

Published Online First 26 March 2013

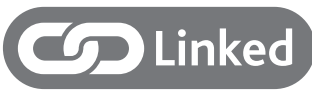

http://dx.doi.org/10.1136/thoraxjnl-2013-203373

Thorax 2013;68:784.

doi:10.1136/thoraxjn-2013-203484

\section{REFERENCES}

1 Sohal SS, Walters EH. Epithelial mesenchymal transition (EMT) in small airways of COPD patients. Thorax 2013:68:783-4.

2 Milara J, Peiró T, Serrano A, et al. Epithelial to mesenchymal transition is increased in patients with COPD and induced by cigarette smoke. Thorax 2013;68:410-20.

3 Lambrecht BN, Hammad $\mathrm{H}$. The airway epithelium in asthma. Nat Med 2012;18:684-92.

4 Shaykhiev R, Otaki F, Bonsu P, et al. Cigarette smoking reprograms apical junctional complex molecular architecture in the human airway epithelium in vivo. Cell Mol Life Sci 2011;68:877-92.

5 Xiao C, Puddicombe SM, Field S, et al. Defective epithelial barrier function in asthma. J Allergy Clin Immunol 2011:128:549-56 e541-512.

6 Sohal SS, Reid D, Soltani A, et al. Evaluation of epithelial mesenchymal transition in patients with chronic obstructive pulmonary disease. Respir Res 2011;12:130.

7 Sohal SS, Reid D, Soltani A, et al. Reticular basement membrane fragmentation and potential epithelial mesenchymal transition is exaggerated in the airways of smokers with chronic obstructive pulmonary disease. Respirology 2010;15:930-8. 